\title{
Filmes e coberturas comestíveis compostas à base de amidos nativos e gelatina na conservação e aceitação sensorial de uvas Crimson
}

\author{
Films and edible coatings based on native starches and gelatin in
} the conservation and sensory acceptance of Crimson grapes

\author{
Farayde Matta FAKHOURI ${ }^{1}$, Luciana Cristina Brigatto FONTES ${ }^{1}$, Priscila Vieira de Macedo GONÇALVES ${ }^{1}$, \\ Cibele Rufato MILANEZ ${ }^{1}$, Caroline Joy STEEL ${ }^{1}$, Fernanda Paula COLLARES-QUEIROZ ${ }^{1 *}$
}

\begin{abstract}
Resumo
Filmes compostos de gelatina com amidos nativos de trigo, sorgo, batata e arroz foram produzidos separadamente e caracterizados quanto às propriedades físico-químicas (solubilidade em água e barreira ao vapor de água), físicas (espessura e opacidade) e mecânicas (resistência à tração e porcentagem de elongação na ruptura). As mesmas soluções filmogênicas foram preparadas e aplicadas em uvas Crimson para avaliação sensorial e acompanhamento da perda de massa durante 22 dias. As coberturas de sorgo e arroz foram as mais eficientes na extensão da vida útil (aumento de 10 dias). Entretanto, em relação aos atributos sensoriais, as uvas com cobertura de arroz não diferiram estatisticamente do controle, que apresentou as menores notas para os parâmetros de aparência global e intenção de compra. O filme de sorgo apresentou uma permeabilidade ao vapor de água de 5,40 g.mm.m².d.kPa, resistência à tração de $85,89 \mathrm{MPa}$, elongação de $6,61 \%$ e opacidade de $40 \%$. Mesmo não apresentando os melhores valores de caracterização, como filme, tornou-se a melhor opção como cobertura. $\mathrm{Na}$ avaliação sensorial, as uvas cobertas obtiveram aceitação igual ou maior que o controle quanto à aparência global, brilho, cor e intenção de compra. Na degustação das uvas, nenhuma das coberturas exerceu influência significativa no aroma, sabor e textura, sendo aceitas pelo consumidor em todos os parâmetros.

Palavras-chave: filme comestível; cobertura comestível; amido nativo; gelatina; uva.
\end{abstract}

\begin{abstract}
Films based on gelatin and native starches from wheat, sorghum, potato and rice were produced separately and characterized as to their physical-chemical (water solubility and water vapor permeability), physical (thickness and opacity) and mechanical (resistance to traction and percentage elongation at rupture) properties. The same solutions were prepared and applied to Crimson grapes for sensory evaluation and determination of weight loss over 22 days. The sorghum and rice coatings were the most efficient in their extending shelf life (a ten day increase). However, with respect to sensory attributes, the grapes with the rice coating did not show a statistical difference when compared to the control, which presented the lowest scores for global appearance purchase. The sorghum coating presented a water vapor permeability of 5.40 g.mm.m-2.d.kPa, resistance to traction of $85.89 \mathrm{MPa}$, stretching of $6.61 \%$ and opacity of $40 \%$. This film did not present the best characterization values, but was the best coating option. In the sensory evaluation, the coated grapes had a better or similar acceptance, when compared to the control, as to global appearance, shine, color and purchase. With respect to the eating characteristics of the grapes, none of the coatings significantly influenced aroma, flavor and texture, and were accepted by the consumers in every parameter.

Keywords: edible film; edible coating; native starch; gelatin; grape.
\end{abstract}

\section{Introdução}

O uso de filmes e coberturas comestíveis em produtos alimentícios parece uma técnica recente, no entanto, a aplicação de ceras em frutas cítricas vem sendo utilizada desde os séculos XII e XIII na China, para retardar a desidratação e melhorar a aparência das mesmas ${ }^{11}$.

Existe um grande interesse no desenvolvimento de biofilmes comestíveis ou degradáveis biologicamente, principalmente devido à demanda por alimentos de alta qualidade, preocupações ambientais sobre o descarte de materiais não renováveis (utilizados como embalagem para alimentos) e oportunidades para criar novos mercados de matérias-primas formadoras de filme, como hidrocolóides e lipídios ${ }^{27,16}$.

Em relação à nomenclatura, a maioria dos pesquisadores usa os termos "filme" e "cobertura" indiscriminadamente. No

\footnotetext{
Recebido para publicação em 14/8/2006

Aceito para publicação em 23/4/2007 (001814)

Departamento de Tecnologia de Alimentos, Faculdade de Engenharia de Alimentos, Universidade Estadual de Campinas - UNICAMP,

Cidade Universitária "Zeferino Vaz", s/n, CEP 13083-970

Campinas - SP, Brasil,

E-mail:fercolla@fea.unicamp.br

*A quem a correspondência deve ser enviada
}

entanto, a cobertura é uma fina camada de material aplicado e formado diretamente na superfície do produto, enquanto que o filme é pré-formado separadamente e aplicado posteriormente sobre o produto $^{19,24}$. Podem ser classificados em comestíveis e/ou biodegradáveis, dependendo dos constituintes utilizados para sua produção e da quantidade das substâncias empregadas $^{30}$.

Os filmes e coberturas possuem a função de inibir ou reduzir a migração de umidade, oxigênio, dióxido de carbono, lipídios, aromas, dentre outros, pois promovem barreiras semipermeáveis. Além disso, podem transportar ingredientes alimentícios como: antioxidantes, antimicrobianos e flavorizantes, e/ou melhorar a integridade mecânica ou as características de manuseio do alimento ${ }^{24}$.

Os biopolímeros mais utilizados na elaboração de filmes e coberturas comestíveis são as proteínas (gelatina, caseína, ovoalbumina, glúten de trigo, zeína e proteínas miofibrilares), os polissacarídeos (amido e seus derivados, pectina, celulose e seus derivados, alginato e carragena) e os lipídios (monoglicerídeos acetilados, ácido esteárico, ceras e ésteres de ácido graxo) ou a combinação dos mesmos ${ }^{10}$. 
Os filmes elaborados a partir de polissacarídeos ou proteínas possuem excelentes propriedades mecânicas, ópticas e sensoriais, porém são sensíveis à umidade e apresentam alto coeficiente de permeabilidade ao vapor d'água. Ao contrário, as coberturas compostas de lipídios apresentam boas propriedades de barreiras ao vapor d'água, mas são opacas e pouco flexíveis, além de apresentarem sabor residual, o que pode influenciar as características sensoriais do alimento. A combinação dos biopolímeros tem como vantagem agregar os pontos positivos de cada um dos constituintes utilizados ${ }^{17}$.

Por estarem em contato com os alimentos, é desejável que os filmes e coberturas comestíveis apresentem propriedades sensoriais neutras (transparente, inodoro e insípido), de modo a não alterar a qualidade dos alimentos ${ }^{18}$.

A utilização de filmes e coberturas comestíveis está relacionada com sua capacidade de agir como um adjunto para promover maior qualidade, estendendo a vida de prateleira e possibilitando a economia com materiais de embalagem final ${ }^{23}$.

Entre os diversos materiais pesquisados para a produção de filmes comestíveis e/ou biodegradáveis, a gelatina merece atenção. A gelatina é uma proteína de origem animal obtida do colágeno por hidrólise ácida ou básica, e amplamente utilizada na indústria alimentícia e farmacêutica. Além disso, a gelatina no Brasil é produzida em abundância, a baixo custo e com propriedades funcionais adequadas para a fabricação de biofilmes ${ }^{7}$.

A gelatina foi amplamente pesquisada tanto na elaboração de filmes como em coberturas comestíveis. SOBRAL ${ }^{31}$, num estudo sobre a influência da espessura nas propriedades de filmes de gelatina, concluiu que a força na ruptura, a permeabilidade ao vapor de água e a cor dos filmes são influenciados linearmente pelo aumento da espessura.

Coberturas filmogênicas elaboradas com gelatina, triacetina e ácido láurico foram aplicadas sobre goiabas brancas e mostraram-se efetivas na extensão da vida útil das goiabas em relação às frutas in natura sem cobertura, sendo bem aceitas pelos consumidores durante 20 dias e sob refrigeração a $12{ }^{\circ} \mathrm{C}^{15}$.

Os filmes à base de amido apresentam boas propriedades mecânicas e excelente propriedade de barreira ao $\mathrm{O}_{2}{ }^{1,29}$. A fécula de mandioca tem sido freqüentemente testada como matéria-prima para este fim, em função da sua transparência e baixo custo ${ }^{8}$. O uso de coberturas de fécula de mandioca nas concentrações de 1, 2, 3, 4 e 5\% em morangos foi eficiente na minimização da perda de massa e aumento de 5 vezes na vida útil da fruta ${ }^{20}$.

Filmes comestíveis têm sido considerados uma alternativa para estender a vida de prateleira de frutas e vegetais, causando efeito semelhante ao da utilização de uma atmosfera modifica$\mathrm{da}^{28}$. O controle da permeabilidade ao vapor de água, oxigênio e dióxido de carbono influencia diretamente a estabilidade do fruto durante a sua estocagem.

As perdas pós-colheita de produtos vegetais são estimadas em $25 \%$ do total produzido. A eliminação ou minimização des- tas perdas apresenta diversas vantagens, como: aumento no abastecimento de produtos sem aumento da área de cultivo, economia energética relacionada à fração de sua produção e comercialização, redução da poluição e satisfação das necessidades do consumidor com a mesma quantidade de energia, terra, água e trabalho ${ }^{9}$.

O objetivo deste trabalho foi o desenvolvimento de filmes compostos elaborados a partir de gelatina e diferentes tipos de amido nativo (arroz, batata, sorgo e trigo), utilizando como plastificante o sorbitol, e sua caracterização em relação à espessura, cor, solubilidade em água, propriedades de barreira (permeabilidade ao vapor de água) e propriedades mecânicas (resistência mecânica e elongação). Posteriormente, as formulações foram utilizadas como coberturas comestíveis no aumento da vida de prateleira de uvas frescas Crimson, mantidas sob refrigeração e umidade relativa controladas durante 20 dias. O estudo da aplicação das coberturas foi realizado com a uva, pois é um fruto frágil com elevado teor de água em sua composição (81\%) e grande superfície de transpiração em relação a sua massa. Tal fruta possui alto valor comercial, sendo grande parte da sua produção voltada à exportação.

\section{Material e métodos}

\subsection{Materiais}

Os materiais utilizados neste trabalho foram: i) gelatina Tipo A (Leiner Davis Gelatin, São Paulo - SP, Brasil); ii) sorbitol (Synth, Diadema - SP, Brasil); iii) amido de trigo (Serrana, Porto Alegre - RS, Brasil); iv) amido de sorgo (Amidos Naviraí, Naviraí - MS, Brasil); v) amido de batata (Metachem, São Paulo - SP, Brasil); vi) amido de arroz (Clariant, São Paulo, SP, Brasil); e vii) uvas Crimson (CEASA, Campinas - SP, Brasil).

\subsection{Metodologia para elaboração dos biofilmes}

A solução de gelatina (GEL) para elaboração dos biofilmes foi obtida hidratando-se $10 \mathrm{~g}$ de gelatina em $100 \mathrm{~mL}$ de água destilada durante 1 hora. Após este período, a solução foi aquecida a $85{ }^{\circ} \mathrm{C}$ por 10 minutos. O sorbitol foi adicionado na concentração de $5 \%$ em relação à massa da gelatina, sob agitação magnética até a sua homogeneização. As suspensões de amido (trigo - TRI, sorgo - SOR, batata - BAT e arroz - ARR) foram preparadas utilizando-se $3 \mathrm{~g}$ de amido em $100 \mathrm{~mL}$ de água destilada e $10 \%$ de sorbitol em relação à massa de amido. A suspensão preparada foi aquecida a $85^{\circ} \mathrm{C}$ em banho-maria por 5 minutos para os amidos de trigo e de sorgo, e por 3 minutos para os amidos de batata e de arroz. Estes tempos foram determinados visualmente em testes preliminares, considerando o tempo necessário para o aumento da viscosidade e a perda de opacidade das suspensões (relacionados à gelatinização do amido). Após o preparo das soluções, foi realizada a mistura da solução de gelatina com cada tipo de amido separadamente, para a formação de quatro diferentes soluções filmogênicas (GEL/TRI; GEL/SOR; GEL/BAT e GEL/ARR). Alíquotas de $20 \mathrm{~mL}$ da solução filmogênica foram distribuídas em placas plaxiglass de $14 \mathrm{~cm}$ de diâmetro e secas a $25^{\circ} \mathrm{C}$ por 24 horas, para a elaboração dos biofilmes. 


\section{Espessura do filme}

A espessura dos filmes foi medida utilizando-se um micrômetro (Modelo MDC-25M, Mitutoyo, MFG, Japão). A espessura foi determinada como sendo a média de cinco medidas aleatórias em diferentes partes do filme.

\section{Permeabilidade ao vapor de água}

A permeabilidade ao vapor de água foi determinada gravimetricamente a $25{ }^{\circ} \mathrm{C}$, de acordo com o método $\mathrm{ASTM}^{2}$. As amostras, triplicatas de cada filme, foram acondicionadas em dessecadores a $25{ }^{\circ} \mathrm{C}$, umidade relativa de $52 \% \pm 2$, por 48 horas antes da medição.

\section{Solubilidade em água}

A solubilidade em água dos filmes foi determinada em triplicata, sendo que amostras dos filmes foram recortadas em discos de $2 \mathrm{~cm}$ de diâmetro. A porcentagem inicial da matéria seca de cada amostra foi determinada em estufa a $105^{\circ} \mathrm{C}$ por 24 horas. Após a primeira pesagem, as amostras foram imersas em recipientes contendo $50 \mathrm{~mL}$ de água destilada, e agitadas lenta e periodicamente por 24 horas e a $25^{\circ} \mathrm{C}$. Após este período, as amostras foram removidas e secas $\left(105^{\circ} \mathrm{C}\right.$ por 24 horas) para determinar a massa da matéria seca que não se dissolveu em água.

\section{Propriedades mecânicas}

A resistência à tração e a porcentagem de elongação na ruptura foram determinadas utilizando o texturômetro TA-XT2 (Stable Micro Systems, Surrey, UK), operando de acordo com o método padrão $\mathrm{ASTM}^{3}\left(25^{\circ} \mathrm{C}\right.$, separação inicial das garras = $50 \mathrm{~mm}$ e velocidade do probe $=100 \mathrm{~mm} / \mathrm{minuto}$ ). Os filmes foram recortados formando corpos de prova de $100 \mathrm{~mm}$ de comprimento e 25,4 mm de largura. Os filmes foram condicionados por dois dias à temperatura ambiente e umidade relativa de $52 \%$ antes da medição.

\section{Opacidade}

A opacidade dos filmes foi determinada utilizando o colorímetro Hunterlab ${ }^{21}$. As determinações foram feitas em triplicata, após a calibração do colorímetro com um fundo padrão branco, cinza e preto. A opacidade foi determinada através da equação:

$\mathrm{Op}=\frac{\mathrm{Opn}}{\mathrm{Opb}} \times 100$

em que:

Op = opacidade do filme $(\%)$

Opn = opacidade do filme sobreposto a um fundo negro

$\mathrm{Opb}=$ opacidade do filme sobreposto a um fundo branco

\subsection{Metodologia para aplicação das coberturas nas uvas}

As uvas Crimson, antes de receberem as coberturas de gelatina e amido, foram imersas durante 30 minutos em uma solução de água e hipoclorito (0,01\%). Após este período, as frutas foram retiradas da solução e mantidas sob temperatura ambiente até secagem completa.

As uvas secas e higienizadas foram divididas em quatro lotes, sendo cada lote imerso durante um minuto em uma solução filmogênica diferente (preparadas de acordo com o item 2.2), e permanecendo sob temperatura ambiente por 12 horas até a secagem completa da cobertura. Para o tratamento controle, as frutas foram preparadas da mesma maneira, mas ao invés da imersão na solução filmogênica, foram imersas em água destilada por 1 minuto.

\section{Perda de massa}

As uvas foram armazenadas sob temperatura de refrigeração de $5 \pm 2{ }^{\circ} \mathrm{C}$ e UR de $60 \pm 2 \%$, sendo pesadas em balança semi-analítica nos dias $1,4,7,10,13,16,19$ e 22, para o cálculo da perda de massa.

\section{Avaliação sensorial}

Para a avaliação da possível interferência da aplicação da cobertura nas características sensoriais da fruta, os consumidores avaliaram cinco diferentes tipos de amostras de uva: i) uva controle; ii) uva com GEL/TRI; iii) uva com GEL/SOR; iv) uva com GEL/BAT; e v) uva com GEL/ARR.

A avaliação sensorial das uvas foi realizada por 40 provadores não-treinados na faixa etária de 25-35 anos. As uvas foram avaliadas 24 horas após a aplicação da cobertura. A ordem de apresentação das mesmas e o número de provadores seguiram o delineamento proposto por MACFIE e BRATCHELL ${ }^{26}$, que considera o balanceamento dos efeitos "first-order e carryover". Os atributos analisados visualmente foram: aparência global, cor, brilho e intenção de compra. Na degustação, os provadores analisaram: aroma, sabor, textura e intenção de compra. As uvas foram servidas monadicamente, codificadas com três dígitos e apresentadas inteiras (bagas individuais) em prato branco sobre mesa também branca.

Nas avaliações sensoriais, os provadores avaliaram o quanto gostaram ou desgostaram das amostras, através de uma escala hedônica de nove pontos com os extremos correspondendo a: "desgostei extremamente" (1) e "gostei extremamente" (9). Somente para a avaliação da "intenção de compra" foi utilizada escala de cinco pontos, na qual os extremos correspondiam a: “certamente não compraria” (1) e "certamente compraria” (5).

\subsection{Análise estatística}

O programa Statistica ${ }^{\circledR}$ (StatSoft, USA) foi usado para calcular as diferenças significativas entre as propriedades de todos os filmes, no intervalo de $95 \%$ de confiança.

\section{Resultados e discussão}

\subsection{Propriedades dos biofilmes compostos}

A espessura dos biofilmes compostos variou de $0,053 \mathrm{~mm}$ (GEL/TRI) a 0,063 mm (GEL/BAT), conforme os dados apre- 
sentados na Tabela 1. A permeabilidade ao vapor de água (PVA) variou de 4,22 a 5,53 g.mm.m-2.d.kPa para os filmes GEL/BAT e GEL/TRI, respectivamente. O biofilme composto GEL/ARR não foi diferente significativamente dos filmes com nenhum outro tipo de amido quanto à permeabilidade ao vapor de água (Tabela 1). Estes valores são semelhantes aos encontrados por TANADA-PALMU, FAKHOURI e GROSSO ${ }^{32} \mathrm{em}$ biofilmes compostos de $20 \%$ de glúten de trigo e $10 \%$ de gelatina $\left(5,29\right.$ g.mm.m $\left.{ }^{-2} \cdot d . \mathrm{kPa}\right)$, e por BATISTA ${ }^{4}$ em filmes compostos de $2 \%$ de pectina e $10 \%$ de gelatina, cujo valor de PVA foi de 4,35 g.mm. $\mathrm{m}^{-2}$.d.kPa, sendo maiores do que os encontrados por KAMPER e FENNEMA ${ }^{22}$ para filmes de hidroxipropilmetilcelulose (1,92 g.mm.m ${ }^{-2}$.d.kPa) e menores que os de celofane $\left(7,27 \text { g.mm. } \mathrm{m}^{-2} \cdot \mathrm{d} \cdot \mathrm{kPa}\right)^{33}$.

Tabela 1. Espessura, permeabilidade ao vapor de água e solubilidade em água dos biofilmes compostos de gelatina e amidos nativos.

\begin{tabular}{cccc}
\hline Filmes & Espessura $(\mathrm{mm})$ & $\begin{array}{c}\text { Permeabilidade } \\
\text { ao vapor de água } \\
\text { (g.mm.m }{ }^{-2} \cdot \text { d.KPa) }\end{array}$ & $\begin{array}{c}\text { Solubilidade em } \\
\text { água }(\%)\end{array}$ \\
\hline GEL/TRI & $0,053 \pm 0,018^{\mathrm{a}}$ & $5,53 \pm 0,02^{\mathrm{a}}$ & $24,38 \pm 2,72^{\mathrm{a}}$ \\
GEL/SOR & $0,056 \pm 0,018^{\mathrm{a}}$ & $5,40 \pm 0,39^{\mathrm{a}}$ & $29,09 \pm 2,69^{\mathrm{a}}$ \\
GEL/BAT & $0,063 \pm 0,002^{\mathrm{a}}$ & $4,22 \pm 0,26^{\mathrm{b}}$ & $27,53 \pm 0,84^{\mathrm{a}}$ \\
GEL/ARR & $0,059 \pm 0,012^{\mathrm{a}}$ & $5,10 \pm 0,01^{\text {ab }}$ & $30,20 \pm 1,19^{\mathrm{a}}$ \\
\hline
\end{tabular}

A solubilidade em água dos biofilmes variou de 24,38\% para GEL/TRI a 30,20\% para GEL/ARR (Tabela 1). Esta variação, porém, não foi diferente estatisticamente $(\mathrm{p} \leq 0,05)$. Estes valores foram semelhantes aos encontrados por FAKHOURI e COLLARES $^{14}$ em biofilmes formados por $10 \%$ de gelatina e $3 \%$ de amido de milho nativo (31,41\%), e superiores aos encontrados por TAYLOR ${ }^{33}$ para biofilmes de $2 \%$ de pectina e $10 \%$ de gelatina (18,33\%). Esta propriedade direciona a aplicação do biofilme como embalagem de produtos alimentícios. Em alguns casos, a sua total solubilização em água pode ser benéfica, como nos produtos semiprontos destinados ao preparo sob cozimento. Entretanto, quando o alimento é líquido ou então exsuda uma solução aquosa, biofilmes de elevada solubilidade não são indicados.

Os biofilmes GEL/TRI e GEL/ARR foram mais resistentes à tração que GEL/SOR e GEL/BAT; este último foi o menos resistente mecanicamente (56,24 MPa) e também o que apresentou menor porcentagem de elongação $(2,86 \%)$ (Tabela 2$)$. Os valores de resistência à tração encontrados foram maiores que os relatados por BRISTON $^{6}$ para o polietileno de alta densidade (17,3-34,6 MPa), e são semelhantes aos encontrados por FAKHOURI, BATISTA e GROSSO ${ }^{12}$ para filmes simples com $10 \%$ de gelatina plastificados com $5 \%$ de triacetina $(93,81 \mathrm{MPa})$.

Em biofilmes compostos com $2 \%$ de pectina e $10 \%$ de gelatina $^{4}$, a elongação foi inferior $(1,64 \%)$ aos resultados encontrados neste estudo, que variou de $2,86 \%$ para o filme GEL/BAT a $8,10 \%$ para GEL/TRI.

Os biofilmes estudados diferiram estatisticamente quanto à característica de opacidade (Figura 1). O filme GEL/SOR apresentou opacidade de 40\%, seguido do elaborado com GEL/BAT. A opacidade do biofilme composto de GEL/TRI foi
Tabela 2. Propriedades mecânicas dos filmes compostos de gelatina e amidos nativos.

\begin{tabular}{lcc}
\hline \multicolumn{1}{c}{ Filmes } & Resistência à tração $(\mathrm{MPa})$ & Elongação $(\%)$ \\
\hline GEL/TRI & $104,62 \pm 7,11^{\mathrm{a}}$ & $8,10 \pm 1,90^{\mathrm{a}}$ \\
GEL/SOR & $85,89 \pm 3,26^{\mathrm{b}}$ & $6,61 \pm 1,94^{\mathrm{ab}}$ \\
GEL/BAT & $56,24 \pm 7,84^{\mathrm{c}}$ & $2,86 \pm 1,20^{\mathrm{b}}$ \\
GEL/ARR & $101,60 \pm 5,66^{\mathrm{a}}$ & $6,38 \pm 1,78^{\mathrm{ab}}$ \\
\hline
\end{tabular}

Médias com letras iguais na mesma coluna não diferem ao nível de $\mathrm{p} \leq 0,05$.

de aproximadamente $10 \%$ e a do GEL/ARR 20\%. Estes valores são menores e maiores, respectivamente, aos encontrados por FAKHOURI, BATISTA, GROSSO, 2003 para biofilmes com $10 \%$ de gelatina (entre 15 e $20 \%$, simples e plastificados com $5 \%$ de triacetina), o que comprova a maior transparência dos biofilmes formados com GEL/TRI e GEL/ARR, quando comparados aos formados por GEL/SOR e GEL/BAT.

A opacidade pode variar em função do teor de amilose dos amidos, pois suas moléculas em solução, devido à linearidade, tendem a orientar-se paralelamente, aproximando-se o suficiente para se formarem pontes de hidrogênio entre hidroxilas de cadeias adjacentes. Como resultado, a afinidade do polímero por água é reduzida, favorecendo a formação de pastas opacas e filmes resistentes ${ }^{34}$. Além do teor de amilose, outros fatores também podem influenciar o grau de opacidade dos biofilmes, tais como: a concentração de lipídios e cinzas do amido utilizado em sua formulação ${ }^{25}$.

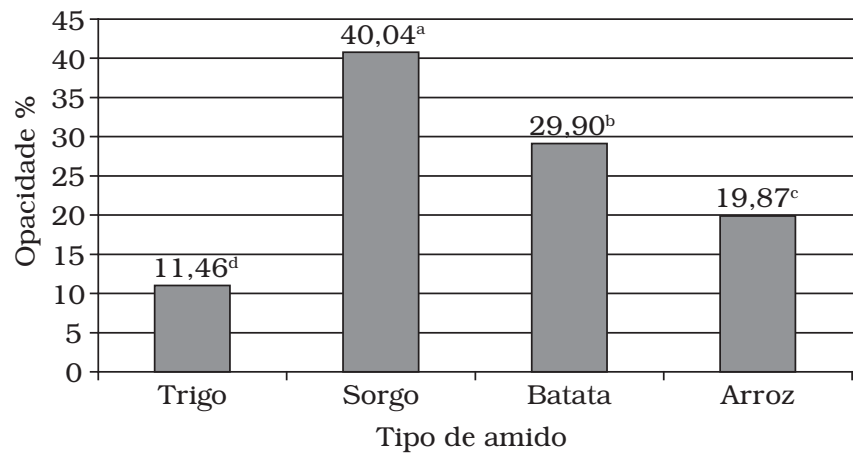

Figura 1. Opacidade dos biofilmes compostos de gelatina e amidos nativos de trigo, sorgo, batata e arroz. Médias com letras iguais não diferem ao nível de $\mathrm{p} \leq 0,05$.

\subsection{Coberturas comestíveis aplicadas em uvas}

\section{Perda de massa das frutas durante armazenamento}

As uvas receberam tratamento com coberturas de gelatina e amidos de diferentes fontes, com a finalidade de avaliar a eficiência de tais coberturas como barreira à perda de água, reduzindo conseqüentemente a perda de massa das frutas durante o armazenamento sob refrigeração.

O tratamento controle apresentou a maior perda de água desde o primeiro ao $22^{\circ}$ dia de armazenamento, quando comparado aos tratamentos que utilizaram coberturas, chegando a perder aproximadamente $14 \%$ de água ao final do experimento (Figura 2). As frutas cobertas com GEL/ARR e GEL/SOR, por 
sua vez, apresentaram uma perda de massa máxima no final do experimento de aproximadamente $8 \%$, não apresentando diferença estatística ao nível de 5\% de significância (Tabela 3). Esta perda foi observada para o tratamento controle no $12^{\circ}$ dia do experimento, resultando em um aumento de vida útil de 10 dias.
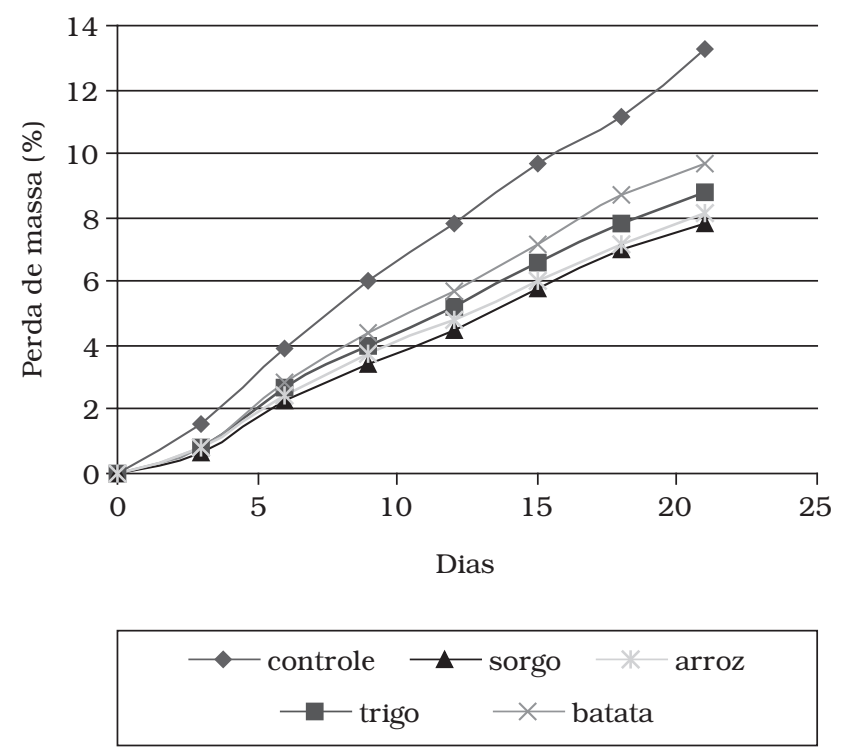

Figura 2. Perda de massa de uvas Crimson armazenadas durante 22 dias sob refrigeração.

Dentre as coberturas estudadas, a maior perda de massa foi observada para as uvas cobertas com GEL/BAT (aproximadamente $10 \%$ ), sendo que o tratamento controle apresentou este valor de perda de água já no $15^{\circ}$ dia de armazenamento, resultando em uma extensão de vida útil de 7 dias. Um estudo utilizando diversas combinações de amido e gelatina na cobertura de uvas, também armazenadas sob refrigeração durante vinte dias, detectou uma extensão na vida útil das uvas de 11 dias, quando o amido de mandioca foi utilizado ${ }^{13}$.

Tabela 3. Valores médios da perda de massa das uvas Crimson após 22 dias de estocagem sob refrigeração.

\begin{tabular}{cc}
\hline Tratamentos & Perda de massa (\%) \\
\hline Controle & $13,24^{\mathrm{a}}$ \\
GEL/TRI & $8,78^{\mathrm{c}}$ \\
GEL/SOR & $8,10^{\mathrm{d}}$ \\
GEL/BAT & $9,67^{\mathrm{b}}$ \\
GEL/ARR & $7,81^{\mathrm{d}}$ \\
\hline
\end{tabular}

Médias com letras iguais não diferem ao nível de $\mathrm{p} \leq 0,05$.

\section{Avaliação sensorial}

Na avaliação dos parâmetros: aparência global, cor, brilho e intenção de compra, para os quatro tipos de tratamentos utilizados, as frutas cobertas com GEL/TRI, GEL/SOR e GEL/BAT apresentaram valores estatisticamente superiores ao tratamento controle em todos os parâmetros avaliados (Tabela 4).

Frutas cobertas com GEL/ARR não diferiram estatisticamente em relação às demais coberturas e também ao tratamento controle quanto à aparência global e intenção de compra. Com isso, pode-se concluir que a melhor cobertura para uva Crimson foi a GEL/SOR, que apresentou a menor perda de massa, não diferindo da cobertura de GEL/ARR, e não sendo estatisticamente igual ao controle nos parâmetros da análise visual.

Tabela 4. Análise sensorial de aparência das uvas cobertas.

\begin{tabular}{ccccc}
\hline Filmes & $\begin{array}{c}\text { Aparência } \\
\text { global }\end{array}$ & Cor & Brilho & $\begin{array}{c}\text { Intenção de } \\
\text { compra }\end{array}$ \\
\hline Controle & $6,07^{\mathrm{c}}$ & $6,12^{\mathrm{c}}$ & $4,90^{\mathrm{b}}$ & $3,42^{\mathrm{b}}$ \\
GEL/TRI & $7,67^{\mathrm{a}}$ & $7,35^{\mathrm{ab}}$ & $7,22^{\mathrm{a}}$ & $4,37^{\mathrm{a}}$ \\
GEL/SOR & $7,27^{\mathrm{ab}}$ & $7,62^{\mathrm{a}}$ & $7,37^{\mathrm{a}}$ & $4,25^{\mathrm{a}}$ \\
GEL/BAT & $7,47^{\mathrm{ab}}$ & $7,77^{\mathrm{a}}$ & $7,42^{\mathrm{a}}$ & $4,22^{\mathrm{a}}$ \\
GEL/ARR & $6,67^{\mathrm{bc}}$ & $6,77^{\mathrm{bc}}$ & $7,02^{\mathrm{a}}$ & $3,75^{\mathrm{ab}}$ \\
\hline \multicolumn{4}{l}{ Médias com letras iguais na mesma coluna não diferem ao nível de $\mathrm{p} \leq 0,05}$.
\end{tabular}

A degustação das uvas controle e cobertas foi realizada com o objetivo de avaliar a possível incorporação de aroma e sabor estranhos, assim como a alteração de textura devido à utilização das coberturas. Quando as frutas foram degustadas, o provador não observou nenhuma diferença significativa em relação ao controle quanto ao aroma, sabor, textura e intenção de compra, o que indica que as coberturas não alteraram as propriedades sensoriais das uvas (Tabela 5).

Tabela 5. Análise sensorial de degustação das uvas cobertas.

\begin{tabular}{ccccc}
\hline Filmes & Aroma & Sabor & Textura & Intenção de compra \\
\hline Controle & $5,72^{\mathrm{a}}$ & $7,02^{\mathrm{a}}$ & $6,97^{\mathrm{a}}$ & $3,97^{\mathrm{a}}$ \\
GEL/TRI & $5,60^{\mathrm{a}}$ & $6,97^{\mathrm{a}}$ & $7,07^{\mathrm{a}}$ & $3,77^{\mathrm{a}}$ \\
GEL/SOR & $5,80^{\mathrm{a}}$ & $7,20^{\mathrm{a}}$ & $7,02^{\mathrm{a}}$ & $3,62^{\mathrm{a}}$ \\
GEL/BAT & $5,75^{\mathrm{a}}$ & $7,32^{\mathrm{a}}$ & $7,22^{\mathrm{a}}$ & $3,80^{\mathrm{a}}$ \\
GEL/ARR & $5,77^{\mathrm{a}}$ & $7,25^{\mathrm{a}}$ & $6,80^{\mathrm{a}}$ & $3,80^{\mathrm{a}}$ \\
\hline
\end{tabular}

Médias com letras iguais na mesma coluna não diferem ao nível de $\mathrm{p} \leq 0,05$.

\section{Conclusão}

As coberturas GEL/SOR e GEL/ARR foram as mais eficientes na extensão da vida útil (10 dias mais que o controle) das uvas Crimson armazenadas sob refrigeração durante 22 dias. Entretanto, na avaliação sensorial, a GEL/ARR não diferiu estatisticamente da amostra controle, que apresentou as menores notas nos parâmetros de aparência global e intenção de compra.

O filme GEL/SOR apresentou uma permeabilidade ao vapor de água de 5,40 g.mm.m². d. kPa, resistência à tração de $85,89 \mathrm{MPa}$, elongação de $6,61 \%$ e opacidade de $40 \%$. Este filme, mesmo não apresentando os melhores valores de permeabilidade, resistência e elongação, tornou-se a melhor 
opção como cobertura. Portanto, nem sempre o melhor filme será a melhor cobertura e vice-versa, pois muitos fatores estão envolvidos quando se aplica o biofilme no fruto, tais como: respiração e maturação.

Na avaliação sensorial, as uvas cobertas obtiveram aceitação igual ou maior que o controle quando a aparência global, brilho, cor e intenção de compra foram considerados. Em relação à degustação das uvas, nenhuma das coberturas exerceu influência no aroma, sabor e textura das frutas, sendo aceitas pelo consumidor em todos os parâmetros avaliados.

\section{Referências bibliográficas}

1. ARVANITOYANNIS, M.; BLANSHARD, J. M. Study of diffusion and permeation of gases in undrawn and unaxially drawn films made from potato and rich starch conditioned at different relative humidities. Carbohydr. Polym., v. 24, n. 1, p. 1-15, 1994.

2. ASTM (American Society for Testing and Materials). Method D 882-83: Standard test methods for tensile properties of thin plastic sheeting. In: Annual book of ASTM standards. Conshohocken, PA, 1980.

3. ASTM (American Society for Testing and Materials). Method E 96: Standard test methods for water vapor transmission of materials. In: Annual book of ASTM standards. Conshohocken, PA, 1980.

4. BATISTA, J. A. Desenvolvimento, caracterização e aplicações de biofilmes a base de pectina, gelatina e ácidos graxos em bananas e sementes de brócolos. Campinas, 2004, 137 p. Dissertação (Mestre em Ciência de Alimentos), Faculdade de Engenharia de Alimentos, Universidade Estadual de Campinas (UNICAMP).

5. BOBBIO, F. O.; BOBBIO, P. A. Introdução à química de alimentos, $3^{\text {a }}$ edição. São Paulo: Ed. Varela, 2001.

6. BRISTON, J. H. Plastic films, $3^{\text {a }}$ edição. New York: John Wiley, Inc., 1988.

7. CARVALHO, R. A. Desenvolvimento e caracterização de biofilmes a base de gelatina. Campinas, 1997, 128 p. Dissertação (Mestre em Ciência de Alimentos), Faculdade de Engenharia de Alimentos, Universidade Estadual de Campinas (UNICAMP).

8. CEREDA, M. P.; Bertolini, A. C.; EvangelistA, R. M. Uso do amido em substituição às ceras na elaboração de "filmes" na conservação pós-colheita de frutas e hortaliças: estabelecimento de curvas de secagem. In: CONGRESSO BRASILEIRO DE MANDIOCA, 7; 1992, Recife. 107p.

9. ChITARRA, M. I. F.; CHITARRA, A. B. Pós-colheita de frutas e hortaliças: fisiologia e manuseio, $1^{a}$ edição. Lavras: UFLA, 1990.

10. CUQ, B.; GONTARD, N.; GUILBERT, S. Edible film and coating as active layers. In: ROONEY, M. L. (Ed.) Active food packaging. London: Blackie Academic \& Professional, 1995. p. 111-142.

11. DEBEAUFORT, F.; QUEZADA-GALLO, J. A.; VOILLEY A. Edible films and coatings: tomorrow's packagings: a review. Crit. Rev. Food Sci., v. 38, n. 4, p. 299-313, 1998.

12. FAKHOURI, F. M.; BATISTA, J. A.; GROSSO, C. Efeito de coberturas comestíveis aplicadas em goiabas in natura (Psidium guajava L.). Desenvolvimento e caracterização de filmes comestíveis de gelatina, triacetina e ácidos graxos. Braz. J. Food Technol., v. 6, n. 2, p. 301-308, 2003.

13. FAKHOURI, F. M.; COLLARES, F. P. Edible coatings based on starch and gelatin for refrigerated grapes. In: PROCEEDINGS
OF THE INTRADFOOD - CONFERENCE EFFoST. 2005, Valencia: Editora Elsevier (Londres), v. 2, p. 1447- 1450.

14. FAKHOURI, F. M.; COLLARES, F. P. Impact of drying conditions on the properties of biofilms based on gelatin and different types of corn and manioc starch. In: CONGRESSO IDS. 2006, in press.

15. FAKHOURI, F. M.; GROSSO, C. R. F. Efeito de coberturas comestíveis na vida útil de goiabas in natura (Psidium guajava L.) mantidas sob refrigeração. Braz. J. Food Technol., v. 6, n. 2, p. 203-211, 2003.

16. FAKHOURI, F. M.; WATANABE, K. M.; BEPPU, M. M.; COLLARES, F. P. Estudo da influência da concentração de proteína em biofilmes de gelatina plastificados com sorbitol. In: SLACA - SIMPÓSIO LATINO AMERICANO DE CIÊNCIA DE ALIMENTOS. 2005, Campinas: disponível em CD-ROM.

17. GALLO, J. A. Q.; DEBEAUFORT, F.; CALLEGARIN, F.; VOILLEY, A. Lipidic hydrophobic, physical state and distribution effects on the properties of emulsion-based films. J. Membr. Sci., v. 180, n. 1, p. $37-46,2000$.

18. GONTARD, N. Films et enrobages comestibles: étude et amélioration des proprietes filmogénes du gluten. Montpellier, 1991, 174p. Thèse (Docteur en «Biochimie, biologie cellulaire et moleculaire - Science des Aliments»), Université Montpellier II.

19. GONTARD, N.; GUILBERT, S. Prolongation of the shelf-life of perishable food products using biodegradable films and coatings. Lebensm. Wiss. Technol., v. 29, n. 1-2, p. 10-17, 1995.

20. HENRIQUE, C. M.; CEREDA, C. M. Utilização de biofilmes na conservação pós-colheita de morango (Fragaria Ananassa Duch) cv IAC Campinas. Ci. Tecnol. Aliment., v. 19, n. 2, p. 231-233, 1999.

21. HUNTERLAB. The color management company. Universal software, version 3. Reston, 1997.

22. KAMPER, S. L.; FENNEMA, O. Water vapor permeability of edible bilayer films. J. Food Sci., v. 49, n. 6, p. 1478-1481, 1485, 1984.

23. KESTER, J. J.; FENNEMA, O. R. Edible films and coatings: a review. Food Technol., v. 40, n. 4, p. 47-59, 1986.

24. KROCHTA, J. M.; MULDER-JOHNSTON, C. Edible and biodegradable polymer films: challenges and opportunities. Food Technol., v. 51, n. 2, p. 61-74, 1997.

25. LIPORACCI, J. S. N.; MALI, S.; GROSSMANN, M. V. E. Efeito do metodo de extração na composição e propriedades de amido de inhame (Dioscorea alata). Semina. Ciências Agrárias, v. 26, n. 3, p. 345-352, 2005.

26. MACFIE, H. J.; BRATCHELL, N. Designs to balance the effect of order of presentation and first-order carry-over effect in hall tests. J Sens Stud, v. 4, n. 2, p. 129-148, 1989.

27. PALMU, P. S. T. Preparação, propriedades e aplicação de biofilmes comestíveis à base de glúten de trigo. Campinas, 2003, 244 p. Tese (Doutorado em Alimentos e Nutrição), Faculdade de Engenharia de Alimentos, Universidade Estadual de Campinas, (UNICAMP).

28. PARK, H. J. Development of advanced edible coatings for fruits. Trends Food Sci. Technol., v. 10, n. 8, p. 254-260, 1999.

29. RINDLAV-WESTLING, A.; STADING, M.; HERMANSSON, A. M.; GATENHOLM, P. Structure, mechanical and barrier properties of amylose and amylopectin films. Carbohydr. Polym., v. 36, n. 2-3, p. 217-224, 1998.

30. SHIH, F. F. Edible films from rice protein concentrate and pullulan. Cereal Chem., v. 73, n. 3, p. 406-409, 1996. 
31. SOBRAL, P. L. J. Propriedades funcionais de biolfilmes de gelatina em função da espessura. Ciência \& Engenharia, v. 8, n. 1, p. 60-67, 1999.

32. TANADA-PALMU, P. S.; FAKHOURI, F. M.; Grosso, C. R. F. Filmes biodegradáveis: extensão da vida útil de frutas tropicais. Biotecnologia: Ciência \& Desenvolvimento, ano 5, n. 26, p. 12-17, maio/junho 2002.
33. TAYLOR, C. C. Cellophane. In: BAKKER, M. (Ed.) The Wiley encyclopedia of packaging technology. New York: John Wiley \& Sons, 1986. p. 159-163.

34. WURZBURG, O. B. Cross-linked starches. In: WURZBURG, O. B. (ed.) Modified starches: properties and uses. Boca Raton: CRC Press, 1986. Cap. 3, p. 41-53. 